\title{
Highly compliant transparent electrodes
}

\section{Citation}

Shian, Samuel, Roger M. Diebold, Alena McNamara, and David R. Clarke. 2012.

“Highly Compliant Transparent Electrodes." Applied Physics Letters 101 (6): 061101. doi:10.1063/1.4742889.

\section{Published Version}

doi: $10.1063 / 1.4742889$

\section{Permanent link}

http://nrs.harvard.edu/urn-3:HUL.InstRepos:25719687

\section{Terms of Use}

This article was downloaded from Harvard University's DASH repository, and is made available under the terms and conditions applicable to Open Access Policy Articles, as set forth at http:// nrs.harvard.edu/urn-3:HUL.InstRepos:dash.current.terms-of-use\#OAP

\section{Share Your Story}

The Harvard community has made this article openly available.

Please share how this access benefits you. Submit a story.

Accessibility 


\title{
HIGHLY COMPLIANT TRANSPARENT ELECTRODES
}

\author{
Samuel Shian ${ }^{1)}$, Roger M. Diebold ${ }^{1,2)}$, Alena McNamara ${ }^{3)}$, David R. Clarke ${ }^{1)}$ \\ ${ }^{1}$ School of Engineering and Applied Sciences, Harvard University, \\ Cambridge, Massachusetts 02138 \\ ${ }^{2}$ Materials Department, University of California, \\ Santa Barbara, California 93106 \\ ${ }^{3}$ Mount Holyoke College, South Hadley, Massachusetts 01075
}

Adaptive optical devices based on electric field induced deformation of dielectric elastomers require transparent and highly compliant electrodes to conform to large shape changes. Electrical, optical, and actuation properties of acrylic elastomer (VHB) electrodes fabricated with single-walled carbon nanotubes (SWCNTs) and silver nanowires (AgNWs) have been evaluated. Based on these properties, a figure of merit is introduced for evaluating the overall performance of deformable transparent electrodes. This clearly indicates that SWCNTs outperform AgNWs. Under optimal conditions, optical transparency as high as $91 \%$ at $190 \%$ maximum actuation strain is readily achievable using SWCNT electrodes. 
Compliant, transparent electrodes are essential for the development of adaptive optical devices actuated using dielectric elastomers. These transparent elastomers are capable of very large strain and shape actuation in response to applied voltages. Although actuators based on dielectric elastomers have already been widely investigated as potential replacements as electromechanical actuators for motors, pneumatic pistons, and solenoid actuators ${ }^{1}$, most researchers have used electrically percolative particle-elastomer composites (e.g., carbon blacks in silicone grease) or corrugated metals film (e.g., silver film by Danfoss Polypower A/S) as electrode materials. ${ }^{2}$ Despite the ease of application and commercial availability, these electrodes are, unfortunately, opaque. Thin metal and traditional transparent electrodes, such as ITO, are unsuitable because they cannot sustain the very large strains, several hundred percent, needed to match the strains attainable with common elastomers, such as the VHB acrylic elastomers (3M Co., St. Paul, MN). In addition to being highly compliant and transparent, the electrodes must also have sufficient electrical conductance to distribute charge evenly, have low mechanical stiffness, and a high cyclic loading endurance.

Several authors have produced electrodes with various degrees of optical transparency. For instance, Yuan et al. demonstrated single-walled carbon nanotube (SWCNT) electrodes that can endure up to $200 \%$ strain and are capable of self-clearing upon local electrical breakdown ${ }^{3}$ but the airbrush application used resulted in translucency due to SWCNT aggregation. ${ }^{4}$ Improved optical transmission was achieved by over-coating the electrodes with an oil but the oil must be contained in any practical configuration. ${ }^{5}$ An alternative approach, implanting metal ions such as gold, palladium, and titanium, has been used to create electrode layers in polydimethylsiloxane capable of maintaining electrical conductance at strains up to $175 \%{ }^{6}$ Unfortunately, at the doses used, the optical transmittance was compromised by the light reflection from the metal, as only $60 \%$ optical transmittance was reported. Similarly, the relatively high density of silver nanowires (AgNWs) used by Yu et al. in their work on bistable electroactive polymers diminished the optical transmittance to below $50 \%{ }^{7}$

In this paper a method for reproducibly forming uniform, transparent and compliant electrodes based on the incorporation of either SWCNTs or AgNWs into a dielectric elastomer sheet is described. To compare the performance of different electrode materials, we introduce a phenomenological-based figure of merit. This figure of merit is useful in determining the 
optimum nanoparticle density to maximize both actuation strain and optical transparency. It is also important since the optical transmittance-electrical actuation response of the electrodes is expected to have a complex dependence on the electro-mechanical properties of the constituent phases as well as the effects of the percolation network on the electrical conductivity, the mechanical stiffness and optical absorption.

We have produced highly compliant and transparent electrode materials (Fig. 1) in a three step process that overcomes previous difficulties and preserves the percolating conducting network. In the first step, stable colloidal suspensions of SWCNTs were prepared and, in the second step, formed into a percolating mat by filtration. In the third step the mat was transferred by contact pressing onto a pre-stretched elastomer sheet. In the initial suspension and dispersion step, $2.5 \mathrm{mg}$ of dry SWCNT (Nano-C, Westwood, MA) were mixed with $45 \mathrm{~mL}$ of $1 \mathrm{wt} \%$ sodium dodecyl sulfate solution in deionized water and then ultrasonicated (Digital 450, Branson, Danbury, CT) for $10 \mathrm{~min}$ at $45 \%$ power to overcome van der Waals forces between nanotube bundles and stabilize individual CNTs sterically through surfactant adsorption. Relatively clear dispersions of SWCNTs were obtained from the supernatant after centrifugation and decanting. Using this method, typical final concentrations of SWCNTs in the solution have been reported to be approximately $0.020-0.025 \mathrm{mg} / \mathrm{mL}{ }^{8}$ The centrifuged dispersion solution was then vacuum filtered through particle-track etched, polycarbonate filters with $50 \mathrm{~nm}$ pore diameters (47 mm diameter, Nuclepore ${ }^{\circledR}$, Whatman, Florham Park, NJ) to form mats of SWCNTs on the filter, matte side facing up. In the third step, the polycarbonate filter was used as a transfer medium to stamp the fiber mat onto a simple dielectric actuator consisting of an elastomer sheet (VHB 4905, 3M Co., nominal thickness $0.5 \mathrm{~mm}$ ) pre-stretched, radially, to 250 $\%$ linear strain and attached to a rigid acrylic support ring with an inner diameter of $39 \mathrm{~mm}$ (Fig. 2a). (In this stretched condition, VHB 4905 exhibits 93\% optical transmittance and has a thickness of $41 \mu \mathrm{m}$ ). Circular electrodes (15 mm diameter) were created on both sides of the stretched VHB sheet by masking the elastomer with laser-cut silicone-lined paper. As the surface of VHB is sticky, the SWCNT mats were easily transferred to the surface of the elastomer by gently pressing the filter onto the sheet. Finally, the filter was released with a few drops of methanol. Electrodes based on AgNW were made the same way except that the suspensions of AgNWs were prepared in ethanol (90 nm average diameter, 20-60 um length, SLW-NW-90, Blue Nano, Charlotte, NC) and then diluted in anhydrous ethanol (200 proof, Sigma Aldrich) to 
$0.5 \mathrm{mg} / \mathrm{mL}$ before filtration. For comparison, electrodes were prepared using thin layers of carbon conductive grease (model 846, MG Chemicals, Burlington, Ontario, Canada).

Representative scanning electron microscopy images of SWCNTs and optical microscope images of AgNW mats on the surface of the elastomer are shown in Fig. 1a and 1b, respectively. As seen in the pictures, the filtration and transfer processes were able to produce relatively uniform nanoparticle distributions throughout the entire electroded area. The density of the nanoparticles could be precisely controlled by varying the volume of the dispersion filtered. Such control can be seen in the range of the optical transmittance achieved as shown in Fig. 1c. Although thick mats of SWCNT could be easily produced by filtration, not all the SWCNTs could then be transferred to the elastomer; thick SWCNT mats tended to separate during transfer, leaving significant quantities of SWCNTs behind on the filter. Consequently, the transfer technique proved limited to producing SWCNT electrodes having more than 50\% optical transmittance. (Fig 1d).

Two sets of in-situ characterization were performed, static characterization of the electrodes in the circular actuator configuration and the other when a voltage was applied to the actuator, stretching the electrodes radially. In-line optical transmittance at $550 \mathrm{~nm}$ was measured using a tungsten filament light source and a spectrometer (model USB 2000, Ocean Optics, Dunedin, FL). Electrical sheet resistance was measured using a customized four-point probe connected to a constant current source (model 6221, Keithley, Cleveland, OH) and a voltmeter (model 2182A, Keithley, Cleveland, OH). To measure the actuation performance, a custom data acquisition program was created using LabVIEW software (National Instruments, Austin, TX). The program integrated a high voltage source (M100DC, ROD-L, Mountain View, CA), a voltmeter (U1200, Agilent, Santa Clara CA), and a USB camera (Dino-Lite, Torrance, CA) to record the electrode diameter. During measurements, the applied voltage was increased at 20 V/s, during which the current flow to the elastomer and the electroded area were continuously monitored until breakdown occurred. In the static conditions, it was found that there is an inverse relationship between nanoparticle density and optical transmittance which follows the LambertBeer law for both SWCNTs and AgNWs (Fig. 1c). In contrast, there is a proportional correlation between the sheet resistance and the optical transmittance of the electrodes until a discontinuity occurs, indicative of a percolation threshold being reached. The percolation thresholds for 
SWCNTs and AgNWs were found to occur at over $91 \%$ and $77 \%$ optical transmittance, respectively (Fig. 1d). It is noteworthy that over much of the conducting region, the sheet resistance of AgNW electrodes is significantly smaller, by approximately two orders of magnitude, than that of SWCNTs having the same transmittance.

The actuation stretch response as a function of applied voltage, $V$, for the AgNW, SWCNT, and carbon grease electrodes up to electrical breakdown are shown in Fig. 2b. Carbon grease was chosen as a reference since its relatively low viscosity (333 to $368 \mathrm{cSt}$ ) and good electrical conductivity $(1.17 \Omega-\mathrm{cm})^{9}$ ensures that the electrode contributes negligible stiffness to the overall device actuation while maintaining excellent electrical conductance. The voltageinduced radial actuation stretch, $\lambda$, is defined as $\lambda=\frac{r}{r_{0}}$, where $r_{0}$ is the initial radius of the electrode area $(7.5 \mathrm{~mm})$ and $r$ is the instantaneous radius during the application of electrical potential (Fig. 2a). The engineering electrical field, $E$, is defined as, $E=\frac{V}{h}=\frac{V}{h_{o}} \lambda^{2}$, where $h$ and $h_{o}$ are the instantaneous and starting thickness of the elastomer in the electroded area, respectively. The plots of actuation stretch as a function of electrical field are shown in Fig. 3. The actuation behaviors of all the electrodes are similar, exhibiting an initial non-linear response, followed by a linear response and then, for lower nanoparticle loadings, a sub-linear response. Each test terminated as a result of electrical rather than mechanical breakdown. The non-linear, high field (above $200 \mathrm{~V} / \mu \mathrm{m}$ ) response with the carbon grease electrodes is an artifact: observations show that over this region the electrode area expands sufficiently to cause the surrounding elastomer to lose tension created by the pre-stretching and buckle out of plane, decreasing the projected area.

At low nanoparticle densities, the maximum attainable actuation stretch is limited by the disruption of the electrically percolative network. When this occurs, the network can no longer effectively distribute charge homogeneously over the elastomer surface. Consequently, the elastomer no longer deforms uniformly, producing local discontinuities as well as local thinning leading to electrical breakdown. At low densities of SWCNTs, the actuation stretch is the same as achieved with carbon grease at low applied fields. However, if electric breakdown does not occur first, the slope deviates from that of carbon grease above a critical field. This is labeled $\lambda_{C}$ in Fig. 3a for the $4.2 \mathrm{mg} / \mathrm{m}^{2}$ SWCNTs. The value of the critical field depends on the starting 
density of the nanoparticles; the higher the starting nanoparticle density the higher the stretch at which the deviation occurs. Since the number of nanoparticles in the electrode remains constant, the density of nanoparticles per unit area decreases as the electrode expands in area. At some stretch value, the nanoparticle density in the electrode falls below the percolation threshold and lower overall actuation results. Empirically, we write the critical stretch at the deviation from carbon grease, $\lambda_{c}$, as:

$$
\lambda_{c}^{2}=\frac{\rho}{\rho_{c}}
$$

where $\rho_{c}$ is the critical nanoparticle density for electrical percolation, and $\rho$ is the initial nanoparticle density. From this relation the critical density of SWCNTs for percolation is calculated to be $2.6 \mathrm{mg} / \mathrm{m}^{2}$ (inset in Fig. 3a). Below this value, the actuation achievable is practically zero, as shown by the $2.3 \mathrm{mg} / \mathrm{m}^{2}$ curve in Fig. 3a.

The data for AgNWs in Fig. 3b indicates that the actuation curves of the AgNW electrodes, while similar to that of carbon grease, occurs at higher fields and shifts to higher fields with higher particle densities. At low particle density, approximately equal volume of AgNW and SWCNT are required to achieve the same optical transmittance. Since the diameter of AgNW is almost two orders of magnitude larger compared to that of SWCNT, i.e.., 90nm vs 1 $\mathrm{nm}$, AgNW coverage over the surface of the elastomer is significantly lower compared to that of SWCNT at the same optical transmittance. For example, at $80 \%$ optical transmittance, the AgNWs and SWCNTs coverage over the elastomer surfaces are approximately $10 \%$ and $100 \%$, respectively. The open area of elastomer between the nanoparticles does not actuate when the voltage is applied, lowering the overall observed stretch reducing the inability of the AgNW electrodes to follow the carbon grease curve. Increasing the density of AgNWs increases both the area coverage and the percolation threshold, raising the maximum actuation stretch. However, at the same time the higher density of nanoparticles also increases the network stiffness, shifting the actuation curve to high fields.

Experimentally, an optimum nanoparticle density for maximum actuation is found. This occurs at approximately 38\% and 72\% optical transmittance for AgNW and SWCNT electrodes, respectively (red dots in Fig. 4). The maximum actuation stretch of AgNWs $\left(\lambda_{\max }=1.6\right)$ is 
smaller than that achievable with SWCNTs $\left(\lambda_{\max }=1.8\right)$ and, as mentioned above, is expected to be controlled by several factors, which include area coverage, percolation threshold and network stiffness.

To quantify the performance of the compliant transparent electrodes, we define a figure of merit (FOM) for actuation:

$$
F O M_{\text {Actuation }}=T^{m}\left(\frac{\varepsilon}{\varepsilon_{S}}\right)_{V_{\text {ref }}}
$$

where $T$ is the percent optical transmittance, the actuation strain $\varepsilon=\lambda-1$, and $\varepsilon_{\mathrm{s}}$ is the strain achieved with carbon grease. Since electrodes are used in pairs, the exponent $m$ is set to 2 for electrodes with identical nanoparticle densities. The FOM is evaluated at the voltage $V_{r e f}$ required to produce the strain, $\varepsilon$. For design purposes, one would select a voltage below the breakdown limit. However, for the purposes of illustration, we choose the worst case scenario corresponding to the strain at electrical breakdown. The FOM of electrodes at various densities along with the maximum possible value at a given transparency (i.e., for carbon grease) is plotted in Fig. 6. This representation clearly shows that SWCNTs have significantly higher maximum FOM compared to AgNWs. The maximum FOM for SWCNT occurs at approximately 91\% optical transmittance with maximum actuation strain of 190\%. Below 85\% optical transparency, the actuation performance of SWCNTs are nearly equivalent to the reference carbon grease electrodes. However, above 91\% optical transmittance, the actuation strain of SWCNT electrodes drops precipitously as the percolation threshold is no longer exceeded uniformly over the electrode area. The FOM of AgNWs is relatively constant from $57 \%$ to $90 \%$ optical transmittance, indicating that as nanoparticle density increases, the gains in the actuation strain are offset by decreases in optical transmittance.

In summary, the interplay between conduction by network percolation and the mechanical stiffness of the fiber-loaded dielectric elastomer indicates that transparent, compliant conducting electrodes can be produced with either SWCNTs or AgNWs. There exist optimum AgNW or SWCNT densities for maximum actuation strains and the figure of merit for actuation for SWCNT electrodes is observed to be significantly higher than that for AgNWs. Despite its 
higher electrical sheet resistance, SWCNT transparent compliant electrodes outperform AgNW electrodes for actuation based optical applications.

Our research was supported by the Harvard MRSEC program of the National Science Foundation under Award number DMR-0820484, the NSF REU program (Alena McNamara) and the Gordon McKay Endowment at Harvard University. 


\section{References}

${ }^{1}$ J. Pons, Emerging Actuator Technologies (John Wiley \& Sons, Ltd, 2005).

${ }^{2}$ F. Carpi, D. De Rossi, R. Kornbluh, R. Pelrine, and P. Sommer-Larsen, Dielectric Elastomers as Electromechanical Transducers: Fundamentals, Materials, Devices, Models and Applications of an Emerging Electroactive Polymer Technology (Elsevier, 2008), p. 344.

${ }^{3}$ W. Yuan, L.B. Hu, Z.B. Yu, T. Lam, J. Biggs, S.M. Ha, D.J. Xi, B. Chen, M.K. Senesky, G. Grüner, and Q. Pei, Advanced Materials 20, 621-625 (2008).

${ }^{4}$ L. Hu, W. Yuan, P. Brochu, G. Gruner, and Q. Pei, Applied Physics Letters 94, 161108 (2009).

${ }^{5}$ W. Yuan, P. Brochu, S.M. Ha, and Q. Pei, Sensors and Actuators A: Physical 155, 278-284 (2009).

${ }^{6}$ S. Rosset, M. Niklaus, P. Dubois, and H.R. Shea, Advanced Functional Materials 19, 470-478 (2009).

${ }^{7}$ S. Yun, X. Niu, Z. Yu, W. Hu, P. Brochu, and Q. Pei, Advanced Materials (Deerfield Beach, Fla.) 24, 1321-7 (2012).

${ }^{8}$ M.J. O’Connell, S.M. Bachilo, C.B. Huffman, V.C. Moore, M.S. Strano, E.H. Haroz, K.L. Rialon, P.J. Boul, W.H. Noon, C. Kittrell, J. Ma, R.H. Hauge, R.B. Weisman, and R.E. Smalley, Science (New York, N.Y.) 297, 593-6 (2002).

${ }^{9}$ MG Chemicals ${ }^{\circledR}$, Carbon Conductive Grease 846 Technical Data Sheet, Retrieved May 13 (2012). 

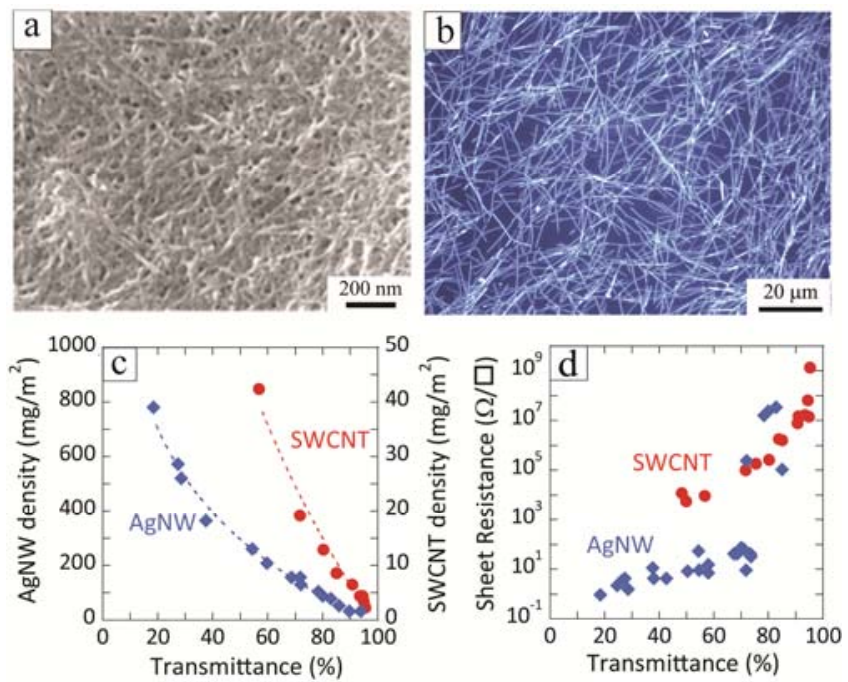

FIG. 1. (a) SEM image of SWCNTs at $62 \mathrm{mg} / \mathrm{m}^{2}$, (b) Optical microscopy image of AgNWs at $160 \mathrm{mg} / \mathrm{m}^{2}$. (c) Optical transmittance at $550 \mathrm{~nm}$ vs. nanoparticle densities. The dashed curves are the Lambert-Beer fit to the data points. (d) Optical transmittance vs. sheet resistance of SWCNT and AgNW electrodes.

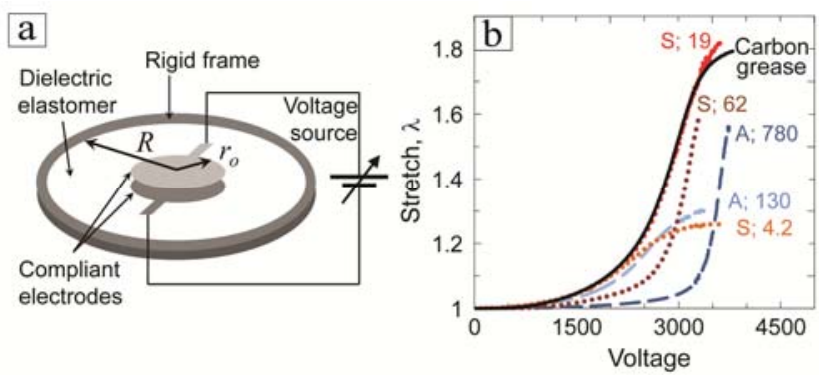

FIG 2. (a) Schematic of circular actuator configuration used to simultaneously record stretch, optical transmittance of the electrodes as a function of applied voltage. (b) Examples of actuation stretch as a function of voltage. The letters A and S refer to AgNW and SWCNT, respectively, and the number is the nanoparticle density in $\mathrm{mg} / \mathrm{m}^{2}$. 

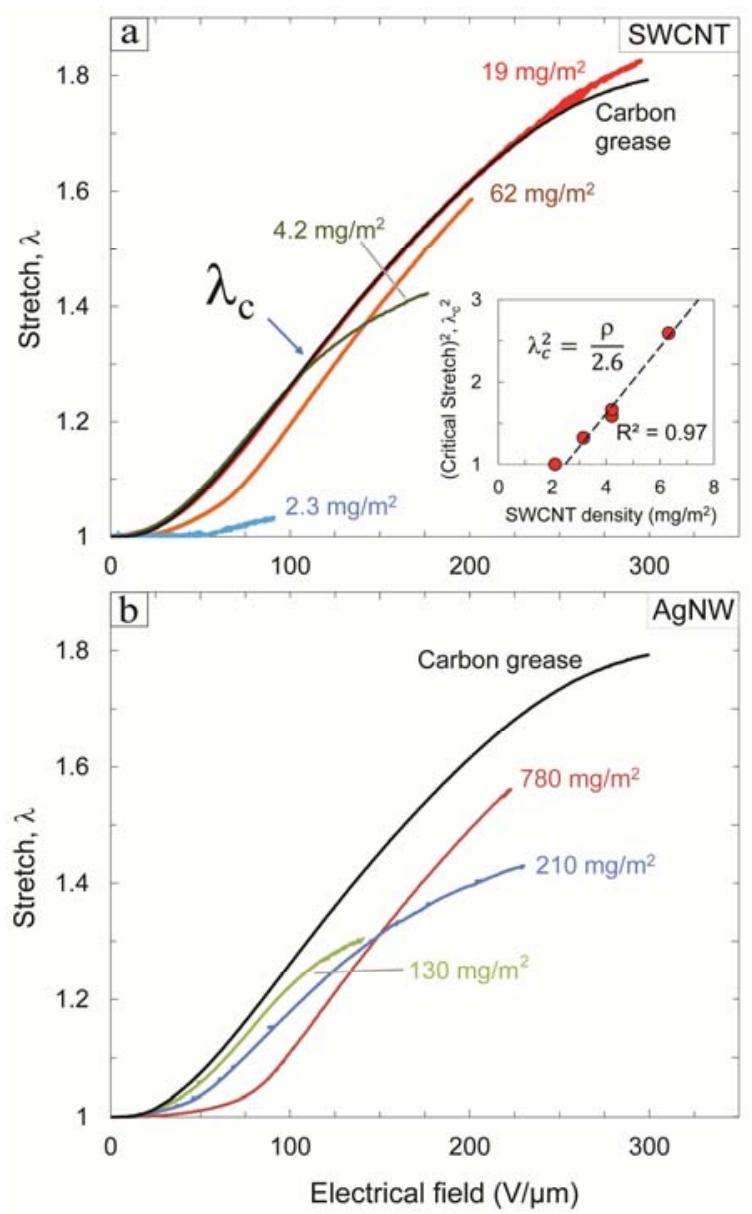

FIG 3. Examples of actuation stretch as a function of electrical field for elastomer electrodes containing (a) SWCNT and (b) AgNW. Inset plot in (a) indicates actuation stretch is limited by the percolation threshold for low optical density SWCNT electrodes.

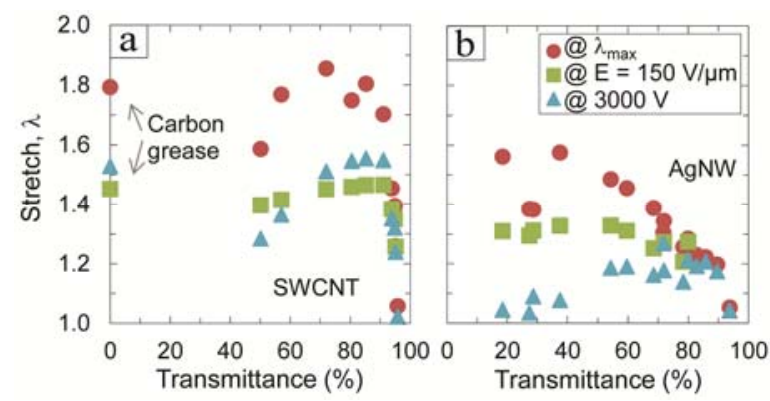

FIG. 4. Actuation stretch as a function of (a) SWCNT and (b) AgNW electrode optical density. Data is shown at a potential of $3000 \mathrm{~V}$ (triangles), at electrical field of $150 \mathrm{~V} / \mu \mathrm{m}$ (squares), and at maximum stretch or breakdown (circles). Data for carbon grease electrodes are shown for comparison. 


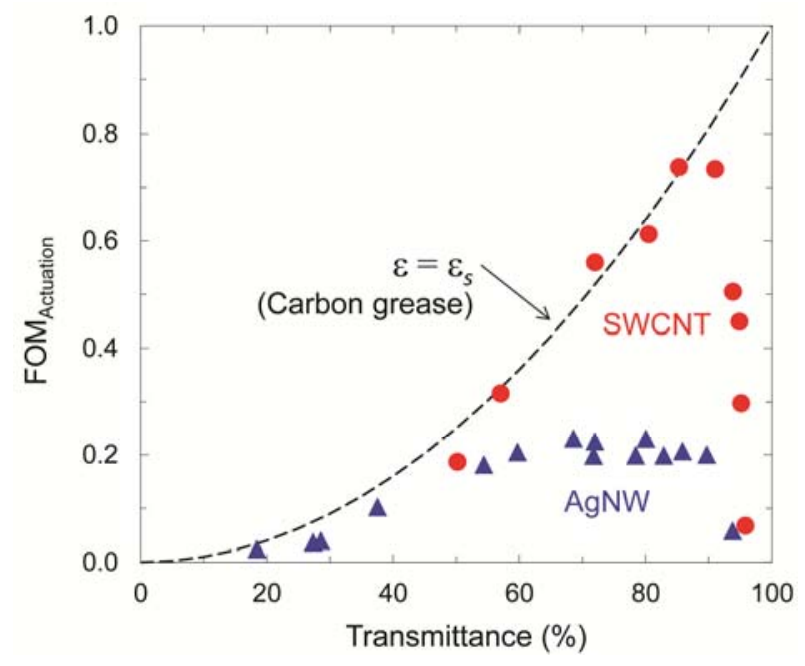

FIG. 5. Figure of merit (FOM) for actuation at various electrode optical densities illustrating that SWCNT electrodes exhibit significantly higher FOM relative to AgNW electrodes. The maximum possible figure of merit is represented by the dashed curve, which is the actuation strain of carbon grease. 